\title{
TERAPIA DIALÉCTICA COMPORTAMENTAL EN UN CASO CON RASGOS DE PERSONALIDAD LÍMITE Y TRASTORNO DE LA CONDUCTA ALIMENTARIA
}

\author{
M. Vicenta Navarro ${ }^{1}$, Mercedes Jorquera ${ }^{2}$, Azucena García-Palacios ${ }^{1,3}, \mathrm{y}$ \\ CRISTINA BOTELLA ${ }^{1,3}$ \\ ${ }^{1}$ Facultad de Psicología, Universidad Jaime I, Castellón de la Plana \\ ${ }^{2}$ Centro Clínico Previ, Valencia \\ ${ }^{3}$ CIBER Fisiopatología de la Obesidad y la Nutrición (CIBEROB), Santiago de Compostela
}

\begin{abstract}
Resumen: El trastorno límite de la personalidad (TLP) presenta una gran heterogeneidad en sus manifestaciones clínicas: desde pacientes con rasgos acusados de TLP, hasta un cuadro completo y/o una gravedad y deterioro importantes. En el grupo de adolescentes, una menor gravedad puede indicar que el TLP está en desarrollo, por lo que intervenir tempranamente podría prevenir la instauración del cuadro completo. El programa de tratamiento psicológico para el TLP con mayor evidencia empírica es la terapia dialéctica comportamental (DBT) creada originalmente por Linehan (1993) para el TLP grave. El objetivo del presente trabajo es describir la aplicación de la DBT en un caso de una mujer joven diagnosticada de bulimia nerviosa y rasgos acusados de TLP. Los buenos resultados preliminares de este estudio indican que una adaptación de la DBT puede ser eficaz y producir mejoras significativas en este perfil de pacientes.
\end{abstract}

Palabras clave: Trastornos de la personalidad, trastorno límite de la personalidad, trastornos de la conducta alimentaria, terapia dialéctico-comportamental.

Dialectical behavior therapy in a case of borderline personality disorder traits and eating disorder

Abstract: Borderline personality disorder (BPD) is heterogeneous in its clinical manifestations: from patients with marked traits of BPD to those that meet full criteria and/or with an important severity and impairment. In adolescents, a lower grade of severity may indicate a developing BPD in which case an early intervention may arrest a further development of the disorder. The psychological treatment program for BPD with the greater amount of empirical evidence is Dialectical Behavior Therapy (DBT) (,b), originally created by Linehan (1993) to treat severe BPD. The aim of this study is to describe the application of DBT in a young woman diagnosed with bulimia nervosa and marked traits of BPD. The good preliminary results of this study indicate that DBT could be efficacious and produce significant improvement in this type of patients.

Keywords: Personality disorders, borderline personality disorder, eating disorders, dialectical behavior therapy.

\section{INTRODUCCIÓN}

El trastorno límite de la personalidad (TLP) se ha definido como un patrón general de ines-

Recibido: 17-marzo-2010; aceptado: 4-mayo-2010

Correspondencia: María Vicenta Navarro Haro, Universidad Jaime I, Departamento de Psicología Básica, Clínica y Psicobiología, Avda. Vicent sos Baynat s/n, 12071 Castellón (España). Correo-e: haro@psb.uji.es

Agradecimientos: Este trabajo ha sido financiado por el Plan de Promoción de la Investigación de la Universidad Jaime I, Fundació Caixa Castelló Bancaixa: Tratamiento psicológico de la personalidad patológica en los trastornos de la conducta alimentaria (P1-1B2005-32) y por CIBER Fisiopatología de la Obesidad y Nutrición. tabilidad en las relaciones interpersonales, en la autoimagen y la afectividad, y una notable impulsividad que comienzan al principio de la edad adulta y se dan en diversos contextos (DSM-IV-TR, APA, 2000). Para diagnosticar este trastorno, el sujeto tienen que cumplir con cinco o más de un conjunto de 9 síntomas (ítems) del DSM-IV-TR. Los rasgos de personalidad constituyen patrones persistentes de formas de percibir, relacionarse y pensar sobre el entorno y sobre uno mismo, que se ponen de manifiesto en una amplia gama de contextos sociales y personales. Según el DSM-IV-TR (APA, 2000), los rasgos de personalidad desa- 
daptativos específicos que no traspasan el umbral para algún trastorno de la personalidad también pueden anotarse en el Eje II.

El TLP presenta una alta comorbilidad con los trastornos de la conducta alimentaria (TCA), principalmente con la bulimia nerviosa (Herzog, Keller, Lavori, Kenny, y Sacks, 1992), oscilando entre el $2 \%$ y el $47 \%$ de los casos (Wonderlich, 1995). Las características esenciales de la bulimia nerviosa consisten en atracones y métodos compensatorios inapropiados para evitar la ganancia de peso. Además, la autoevaluación de los individuos se encuentra excesivamente influida por la silueta y el peso corporales. Para poder realizar un diagnóstico, los atracones y conductas compensatorias inapropiadas deben producirse al menos en un promedio de dos veces a la semana durante un periodo de 3 meses (DSM-IV-TR, APA, 2000).

A pesar de que los trastornos de la personalidad se diagnostican principalmente en la edad adulta, se están realizando estudios en los que se observa la relación entre determinados factores de vulnerabilidad y de protección presentes en la adolescencia (p.ej. aceptación e implicación parental) y la aparición temprana de psicopatología de eje I y de eje II (p.ej. Iglesias y Romero, 2009; Oliva, Giménez, Parra y Sánchez-Queija, 2008). Respecto al TLP, emerge durante la adolescencia (Zanarini et al., 2001), el diagnóstico de este trastorno en este grupo de edad, hasta el momento, no tiene un gran reconocimiento por parte de los clínicos. Según el DSM-IV-TR (APA, 2000), para diagnosticar un trastorno de la personalidad en un sujeto de menos de 18 años, las características deben haber estado presentes durante al menos 1 año y en los casos relativamente raros en los que los rasgos de personalidad desadaptativos particulares del individuo parezcan ser tendentes a extenderse, a persistir y a no limitarse a una etapa particular del desarrollo o a un episodio de trastornos de Eje I. En esta línea, existen estudios empíricos que indican que los criterios diagnósticos para el TLP son tan válidos y estables antes de los 18 años como en la edad adulta (Chanen, Jovev y Jackson, 2007; Chanen et al., 2004; Westen, Shedler, Durrett, Glass y Martens, 2003). Además, los adolescentes con TLP han mostrado tener una discapacidad fun- cional mayor que los adolescentes con otros trastornos de la personalidad (TP) en medidas de funcionamiento social, problemas de rendimiento y escolares, síntomas psiquiátricos y conducta antisocial (Bernstein et al., 1993).

Sobre el pronóstico, se sabe que los predictores más importantes de continuación de los síntomas de trastornos de la personalidad son una elevada gravedad y una edad más joven de inicio. Además, existen hallazgos que indican que los factores de riesgo psicosociales observados en adolescentes y adultos parecen ser también los mismos (Paris, 2005).

Estos datos reflejan la necesidad de poner en marcha programas de prevención e intervención tempranas empíricamente validadas para personas que muestran rasgos acusados o están comenzando a desarrollar un TLP, teniendo en cuenta también que los rasgos de TLP en adolescentes muestran una considerable flexibilidad y maleabilidad.

Por otro lado, algunos trabajos han encontrado que los pacientes con bulimia nerviosa que presentan trastornos de personalidad mantienen una mayor frecuencia de atracones, vómitos y síntomas ansioso-depresivos, mayores dificultades de integración social y un mayor número de intentos de suicidio (p.ej. Matsunaga, Kiriike, Nagata. y Yamagami, 1998; Steiger y Stotland, 1996). Por este motivo, desde las organizaciones dedicadas a diseñar guías clínicas para los trostornos mentales como el National Institute of Clinical Excellence (NICE, 2004) o la American Psychological Association (APA, 2006) se recomienda que los clínicos utilicen tratamientos empíricamente validados para tratar el trastorno alimentario y que incluyan intervenciones focalizadas en el rasgo en aquellos pacientes que presenten patología de la personalidad.

Tradicionalmente, el TLP se ha considerado un trastorno intratable, sin embargo, la publicación de estudios controlados, está abriendo recientemente un camino prometedor en este campo (ver Paris, 2009 para una revisión). Entre las técnicas de tratamiento con mejores resultados encontramos a la terapia dialécticocomportamental (dialectical behavior therapy, DBT) (Linehan, 1993a,b) que ha mostrado ser eficaz en mejorar aspectos claves del TLP (p.ej. 
Linehan et al., 2006; van den Bosch, Koerter, Stijnen, Verheul y van den Brink, 2005; Verheul et al., 2003; Linehan et al., 2002).

La DBT aborda el problema de personalidad del paciente con TLP. Es un programa de tratamiento que se enmarca en la terapia de conducta. El objetivo fundamental del tratamiento es que el paciente aprenda a modular la emocionalidad extrema, reduciendo las conductas desadaptativas dependientes del estado de ánimo, y que aprenda a confiar y validar sus propias experiencias, emociones, pensamientos y comportamientos. Sin embargo, una de las aportaciones principales de esta terapia, es un cambio en el enfoque de la misma. La terapia cognitivocomportamental se centra en conseguir la resolución de problemas emocionales a través del cambio comportamental y cognitivo, y la DBT pone el énfasis en la aceptación y la validación, para desde ahí conseguir el cambio. Según el modelo de Linehan, los individuos con este trastorno han sufrido la invalidación por parte del ambiente, recibiendo el mensaje de que su forma de pensar, comportarse y sentir es errónea y que tienen que cambiar. Cuando se presenta un tratamiento cognitivo-comportamental a este tipo de pacientes se corre el riesgo de que interpreten la base lógica del tratamiento como el mensaje que siempre han escuchado por parte de su ambiente. Esto puede provocar que el paciente adopte una actitud defensiva respecto a la terapia. Según la terapia de Linehan, sólo desde la aceptación, será posible el cambio.

Quizás, la combinación de una terapia focalizada en el problema alimentario con una terapia centrada en los problemas estructurales de la personalidad, podría resultar muy útil en pacientes que presentan este tipo de comorbilidad.

La DBT está siendo adaptada para el tratamiento de otros problemas mentales (p.ej. Lynch, 2000; Telch, Agras y Linehan, 2001). El foco principal de esta terapia son los problemas en la regulación emocional. Siguiendo esta lógica, algunos investigadores estamos estudiando la eficacia de este programa de tratamiento en otros trastornos donde aparecen dificultades en la regulación de los afectos, que en el caso que vamos a presentar sería la bulimia nerviosa. Nuestra experiencia y conocimientos en el tratamiento de los trastornos alimentarios nos lle- va a entender la patología alimentaria como una manifestación de las dificultades en la regulación del afecto dentro de la patología límte de la personalidad.

El objetivo principal del presente trabajo es, por tanto, presentar datos de la eficacia preliminar de un programa de tratamiento basado en la DBT para un caso de una mujer joven diagnosticada de trastorno de la conducta alimentaria y que presenta rasgos acusados de TLP. Con la aplicación de este programa se pretende mejorar significativamente los aspectos cínicos más relacionados con los comportamientos impulsivos propios de la patología límite de esta paciente: uso de sustancias, atracones, vómitos, conductas sexuales de riesgo y conductas autoagresivas y heteroagresivas. Se espera también disminuir la patología emocional y mejorar la regulación del afecto: sintomatología depresiva, ansiedad, expresión de la ira y comer emocional. Y por último, se espera mejorar el nivel de adaptación: nivel de funcionamiento global, resilencia y calidad de vida. Este trabajo se enmarca en un estudio más amplio cuyo objetivo es realizar un ensayo clínico controlado para validar la DBT en población española que padezca TLP y TCA. La participante en este trabajo firmó un consentimiento informado expresando su conformidad.

\section{MÉTODO}

\section{Participante}

La participante de este estudio es una de las pacientes que solicitaban ayuda en el Centro de Psicología PREVI de Valencia, un centro clínico con amplia experiencia en la investigación y tratamiento de los trastornos de la conducta alimentaria y los trastornos de la personalidad. A continuación, se presentan las características más relevantes de la historia clínica de la participante.

Motivo de consulta. La paciente es una mujer de 20 años, está cursando estudios universitarios y su estado civil es soltera. Acude a PREVI debido a la presentación de sintomatología alimentaria de tres años de evolución. 
Descripción del problema. En el momento de evaluación, presenta una dieta restrictiva (que ella identifica con comer sano) que se acompaña de atracones por las tardes, los cuales, en la mayoría de las ocasiones son subjetivos y derivan en vómito autoprovocado. Desde hace unos meses el problema se ha intensificado, presentando, al memos, un vómito diario. No hay una pérdida de peso significativa, presentando un índice de masa corporal (IMC) de 21,5. Refiere experimentar preocupación excesiva acerca de la comida ("todo el día pensando en ella") y el peso, y una sobrevaloración del físico.

Historia familiar. Es la mediana de tres hermanos (sus dos hermanos son varones). Según refiere, el hecho de haber crecido entre dos varones la ha condicionado mucho, ya que sus hermanos han gozado de más libertades que ella. Asimismo, asegura que en casa han recibido una educación muy estricta y rígida por parte del padre. Él ha sido quien ha ejercido la autoridad en casa y todos, incluída la madre, según refiere la paciente, "obedecían”. Describe a su padre como: luchador, racional, autoritario, rígido, emocionalmente frío, desconfiado y "clasista" de cara a sus hijos, y a su madre como: fuerte pero "hacia dentro" (es como una "mosquita", "parece que ni siente ni padece, pero la ves remontar sin ayuda de nadie").

Evolución del problema. Respecto al problema alimentario, la paciente sitúa el inicio a los 14 años, coincidiendo con su desarrollo físico. Recuerda que a esa edad ella empezó a "echar caderas" y subir de peso, de manera que comenzó a obsesionarse con el físico. No manifestaba ningún tipo de manipulación con la comida, sí recuerda que, posteriormente (con 15 años aproximadamente) vomitó por primera vez de forma voluntaria, pero sin intención de adelgazar: tras un cumpleaños, se sintió empachada y vomitó para encontrarse mejor. A raíz de ese día, empezó a emplear el vómito de forma muy aislada, pero cada vez con más frecuencia, como un método para comer lo que le apetecía sin subir de peso. Comenta que, de forma paralela, se iba obsesionando cada vez más con el peso y se habituó a vomitar, al menos una comida al día, comprobando que poco a poco iba bajando de peso. A lo largo de 9 meses aproximadamente, perdió 7 kilos $(\mathrm{IMC}=20)$, pérdida por la que recibió mucho refuerzo social. Sin embargo, durante las vacaciones de verano, recuperó algo de peso. No le molestó ese incremento, lo único que quería era no pasar del límite que ella se había propuesto, ni llegar al peso premórbido (62 kilos). Desde entonces y hasta la actualidad, ha alternado periodos de gran restricción alimentaría con períodos de más normalidad, que, según indica ella misma, dependen mucho de cómo se encuentre su estado de ánimo.

Según refiere la paciente, su principal problema no es la comida, sino su estado emocional que es muy cambiante e intenso. Atribuye sus problemas emocionales a un hecho que la marcó, la muerte de su tío paterno. Comenta que cuando ella tenía 16 años, su tío se suicidó. La familia estaba muy unida a él porque era soltero y pasaba mucho tiempo con ellos en casa. Desconocen el motivo por el que lo hizo, al menos, a ella, no le han dado una explicación. Nunca se ha hablado en casa del tema, se convirtió en un tabú, y los padres invalidaban la expresión emocional de sus hijos (no se permitía llorar a nadie) ante cualquier signo de duelo. Por ese motivo, la paciente percibe que todos han salido hacia delante, pero ella se ha quedado estancada. Siente estar enfadada con su tío por lo que hizo y con su padre porque, a raíz de esto, la relación con él ha empeorado mucho y, ella lo achaca a que él tampoco ha superado la muerte de su hermano.

Con respecto a la patología de la personalidad, refiere labilidad emocional desde niña, lo que le causaba muchos problemas en casa, ya que la expresión emocional no estaba permitida. Este patrón familiar se acentuó tras la muerte de su tío. También presenta conductas impulsivas que comenzaron a aparecer después de la muerte de su tío y que han ido haciéndose más frecuentes y problemáticas. Se trata de conductas autoagresivas y heteroagresicas (p.ej. golpearse, tirar objetos), abuso de alcohol sobre todo los fines de semana y conductas sexuales de riesgo (tener relaciones sexuales con desconocidos sin preservativo, pese a haber recibido educación sexual y conocer los riesgos). Estas conductas las utilizaba como forma de descarga emocional ante las discusiones con su padre o con otras personas (amigos, parejas). 
Además, presentaba dificultades en las relaciones interpersonales. Hace cosas que atentan contra el respeto por sí misma o por los demás con el fin de sentirse aceptada o querida (sobre todo por los chicos). Este patrón con los chicos le hace tener problemas con sus amigas, lo que le lleva a rupturas prematuras y dificultades para conseguir relaciones de amistad estables. Con respecto a su identidad, dice tener la sensación de no encajar con los demás ni con el mundo y refiere sentimientos de vacío en muchas ocasiones. Hasta el momento, no ha recibido nunca tratamiento psicológico ni farmacológico.

En el momento de la evaluación, la paciente cumple los siguientes criterios diagnósticos (DSM-IV-TR):

Eje I: 307.50 Bulimia nerviosa.

Eje II: Z03.2. Sin diagnóstico, rasgos de personalidad límite (V71.09)

Eje III: Sin diagnóstico

Eje IV: Problemas relativos al grupo primario de apoyo y el ambiente social.

Eje V: EAAG: 50 (síntomas graves o cualquier alteración grave en la actividad social, laboral o escolar).

\section{Instrumentos de evaluación}

La evaluación tradicional del TLP se ha centrado sobre todo en la valoración de los criterios diagnósticos y en las conductas más disruptivas que presentan estas pacientes (p.ej., intentos de suicidio, conductas autolesivas, abuso de sustancias). Sin embargo, no existen instrumentos específicos para valorar los problemas emocionales de estos pacientes. Uno de nuestros objetivos es explorar la utilización de medidas de autoinforme que pensamos que pueden tener importancia en este trastorno. En la evaluación para esta paciente, hemos incluido: una serie de instrumentos para diagnosticar la patología límite y la bulimia nerviosa, una medida clínica para recabar información sobre el estado clínico de la paciente y varias medidas de autoinforme para evaluar la psicopatología y nivel de adaptación de la participante. Nos ha interesado sobre todo incluir algún cuestionario que mida estrategias de regulación emocional, ya que es un aspecto clave en la psicopatología del TLP.

Para evaluar el diagnóstico de rasgos de trastorno límite se administró a la paciente la entrevista clínica estructurada para los trastornos de personalidad del Eje II del DSM-IV, SCID-II (First, Gibbon, Spitzer, Williams y Benjamin, 1999) y para delimitar el diagnóstico de bulimia nerviosa se utilizó la Entrevista Clínica de los Trastornos alimentarios (Perpiñá, Botella y Baños, 2006).

Para la evaluación de la patología de la personalidad se utilizaron las siguientes medidas: (1) Inventario de Información Clínica Relevante: se diseñó para esta investigación con el fin de recabar información clínica relevante del estado clínico del paciente (diagnóstico multiaxial, frecuencia de ingresos hospitalarios, intentos de suicidio, frecuencia de conductas impulsivas, uso de sustancias) y fue completado por el terapeuta. (2) Inventario para la Depresión de Beck Segunda Edición (Beck Depression Inventory-II, BDI-II; Beck, Steer y Brown, 1996, versión española validada por Sanz, Navarro y Vázquez, 2003): evalúa la gravedad/ intensidad de los síntomas depresivos. (3) Cuestionario de Ansiedad Estado-Rasgo (State Trait Anxiety Inventory, STAI; Spielberger, Gorsuch, Lushene, Vagg, y Jacobs, 1983, versión española validada por Conde y Franch, 1984). En este trabajo sólo se incluyó la escala de ansiedad rasgo: ansiedad experimentada en un momento concreto. (4) Cuestionario de Afecto Positivo y Negativo (Positive and Negative Affect Schedule, PANAS; Watson, Clark y Tellegen, 1988, versión española validada por Sandín et al., 1999): informa sobre el grado de afectividad positiva y negativa en el individuo. (5) Inventario de Expresión de la Ira EstadoRasgo (STAXI-2; Miguel-Tobal, Casado, CanoVindel y Spielberger, 1999): mide la experiencia y expresión de la ira en tres subescalas: rasgo de la ira $(\mathrm{R})$, estado de la ira $(\mathrm{E})$ e índice de expresión de la ira (IEI); en esta investigación utilizamos este último índice. (6) Escala de Comer Emocional (Emotional Eating Scale; Arnow, Kenardy y Agras, 1995). Esta escala no está validada en castellano. Mide la conducta de comer como una respuesta a estados emocionales. (7) Cuestionario de Regulación Emocional (Emotion Regulation Questionnaire; 
Gross y John, 2003). Esta escala tampoco está validada en castellano. Evalúa estrategias de regulación emocional divididas en dos dimensiones: supresión (suppression): grado con que se tiende a inhibir la expresión de las emociones en respuesta a estresores, y reevaluación (reappraisal): grado con que se intenta pensar sobre las situaciones de forma diferente para cambiar lo que están sintiendo. (8) Escala de Resiliencia de 15 items (Resilience Scale 15, RS15; Wagnild y Young, 1993. Mide la resiliencia psicológica referida a la capacidad para sobrevivir a estresores vitales, crecer y dar significado a los retos. Es una versión corta de la Escala de Resiliencia Psicológica de Wagnild y Young, 1993). (9) Índice de Calidad de Vida (Mezzich, et al. 2000, versión española validada por Bulacio et al., 2004): representa diez aspectos relevantes para la evaluación de calidad de vida y una valoración global de la calidad de vida.

\section{Diseño experimental}

Consiste en un estudio de caso con tres momentos de evaluación pre (antes del tratamiento), post (después del tratamiento) y seguimiento a los 6 meses de finalizada la intervención. Los estudios de caso constituyen una modalidad metodológica de interés en la investigación psicopatológica, ya que aportan un importante conocimiento básico descriptivo que es imposible obtener a partir de los diseños con amplio número de sujetos.

\section{Tratamiento}

El programa de tratamiento psicológico fue la terapia dialéctico-comportamental (Linehan, 1993a). La intervención completa se estructura en tres fases: 1) pre-tratamiento: se establece la relación terapéutica, las metas terapéuticas y los acuerdos entre paciente y terapeuta; 2) primera fase del tratamiento: tiene una duración de un año y se llevan a cabo las metas principales del tratamiento utilizando terapia individual, terapia de grupo, y consultas telefónicas, que se utilizan para ayudar al pacien- te a generalizar las habilidades y estrategias aprendidas en terapia a la vida cotidiana; 3) segunda fase del tratamiento: dedicada al tratamiento del estrés postraumático pero sin duración determinada. Incorpora las técnicas cognitivo-comportamentales diseñadas por Foa y Rothbaum (1998) ya que es frecuente encontrar experiencias traumáticas en las historias de estos pacientes y/o dificultades en la elaboración de acontecimientos traumáticos o pérdidas. 4) tercera fase de tratamiento: no tiene una duración específica, consistiría en que el paciente comience a plantearse y perseguir metas vitales realistas en distintas áreas como el trabajo, la familia, la pareja, etc. Actualmente, sólo se ha publicado la estructura de este terapia hasta la primera fase de tratamiento.

La estructura, las técnicas y el proceso terapéutico están diseñados con el objetivo de tratar las áreas problemáticas propias del trastorno: los problemas de inestabilidad emocional, las dificultades en las relaciones interpersonales, las conductas suicidas e impulsivas, la rigidez cognitiva y las alteraciones en la identidad. Para ello, incluye distintos modos de terapia, siendo la terapia individual la base del programa pero ayudándose de terapia grupal para el entrenamiento en habilidades, consultas teléfonicas para la generalización de las mismas y reuniones del equipo clínico para el seguimiento del caso (García Palacios, 2006).

La terapia grupal sigue las pautas tradicionales de un programa cognitivo-comportamental. Existe un manual de tratamiento que especifica los aspectos a tratar en cada módulo del entrenamiento en habilidades (Linehan, 1993b). Sin embargo, la terapia individual no está basada en un manual. Lo que guía esta modalidad de terapia es una jerarquía de metas terapéuticas que se abordan en función de su importancia. La jerarquía que se establece en la terapia individual es la siguiente: (1) conductas suicidas y parasuicidas; (2) conductas que interfieran con la terapia; (3) conductas que interfieran en la calidad de vida; (4) aumentar habilidades comportamentales. Los objetivos terapéuticos específicos para cada paciente se organizan en función de esa jerarquía de prioridades. En la fase de pretratamiento, las metas terapéuticas se 
organizan siguiendo esta jerarquía. Del mismo modo, el listado de prioridades ayuda al terapeuta y al paciente a centrar los objetivos en cada sesión. La estructura de la DBT permite que exista un orden pre-establecido respecto a los temas a tratar en la sesión.

El objetivo de la terapia grupal es el entrenamiento en cuatro habilidades fundamentales (atención plena, regulación emocional, tolerancia al malestar y eficacia interpersonal) con el fin de mejorar patrones comportamentales, interpersonales, emocionales y cognitivos que causan malestar y que producen una gran interferencia en la vida del individuo. Las habilidades se presentan en el grupo y se practican en el grupo y en la terapia individual. El grupo de terapia se compone de entre seis a ocho pacientes y dos terapeutas, que se reúnen una vez por semana en sesiones de alrededor de dos horas y media (Linehan, 1993b).

La participante de este trabajo recibió 6 meses de DBT individual y grupal. En la terapia individual se conservó el formato estándar de la DBT (Linehan, 1993a) y se incluyeron técnicas cognitivo-comportamentales focalizadas en el tratamiento de la patología alimentaria para tratar el trastorno de Eje I.

En el entrenamiento en grupo se realizó una adaptación ampliando el formato original a 24 sesiones a partir de las indicaciones del manual para el módulo de entrenamiento en habilidades de Linehan (1993b).

\section{Terapia individual}

Consistió en una sesión semanal de 60-90 minutos de duración con un terapeuta especializado en trastornos alimentarios y trastornos de la personalidad con formación en DBT. A continuación, exponemos un resumen del programa de tratamiento individual que se llevó a cabo para esta participante.

El pre-tratamiento se centró en la realización de un análisis de las principales áreas de funcionamiento afectadas en la paciente. La principal fuente de malestar era la inestabilidad afectiva (tristeza, rabia/odio, ansiedad, culpa, alegría) que causaba una desregulación en diferentes áreas como:
1. Identidad: sentimientos de vacío, ineficacia, indefensión aprendida, confusión y distorsión de la imagen corporal.

2. Cognitiva: rigidez cognitiva, pensamiento caracterizado por pensamientos de todo o nada; pensamientos de devaluación: "mi padre no confía en mí, soy una cría"; ideas sobrevaloradas respecto al cuerpo/peso/comida: "debo mantener este peso para gustarle a los chicos".

3. Comportamental: impulsividad extrema: atracones seguidos de vómitos autoinducidos, abuso de alcohol, conductas sexuales de riesgo y conductas agresivas en respuesta a frustraciones.

4. Interpersonal: relaciones de pareja inestables, discusiones con el padre.

Los objetivos que se llevaron a cabo para reducir la desregulación emocional, cognitiva, conductual, interpersonal y de la identidad durante el tratamiento individual fueron acordados por el terapeuta según la siguiente jerarquía:

Meta 1. Reducir conductas parasuicidas: Manifiesta deseo de morir, conductas autoagresivas (darse golpes en distintas partes del cuerpo), conductas heteroagresivas (tirar objetos).

Meta 2. Reducir conductas que interfieran en la terapia: Realización del diario DBT.

Meta 3. Reducir conductas que interfieran en la calidad de vida del paciente: Conductas sexuales de alto riesgo (con desconocidos y sin preservativo), área social (discusiones con el padre), abuso de sustancias (alcohol), y problema alimentario (idea sobrevalorada sobre la imagen, vómitos y atracones).

Después de delimitar las metas a conseguir, el terapeuta confeccionó el diario DBT para la paciente. Este diario servía para concretar los aspectos más importantes a tratar en cada sesión. La paciente rellenaba el diario durante la semana. De este modo, en función de la jerarquía de objetivos, el aspecto que había sido más problemático durante la semana se analizaba con la paciente a través de un análisis funcional 
en el que se relacionaba la situación problemática con el patrón de funcionamiento límite. El siguiente paso consistía en intervenir utilizando distintas estrategias terapéuticas según la meta elegida. Se planteaban los problemas desde una perspectiva de solución de problemas y se en- trenaba a la paciente en distintas técnicas para afrontar esos problemas. En la Figura 1, presentamos un ejemplo de uno de los análisis funcionales que se realizaron con esta paciente. Se trata de una situación problemática que se repetía con bastante frecuencia:

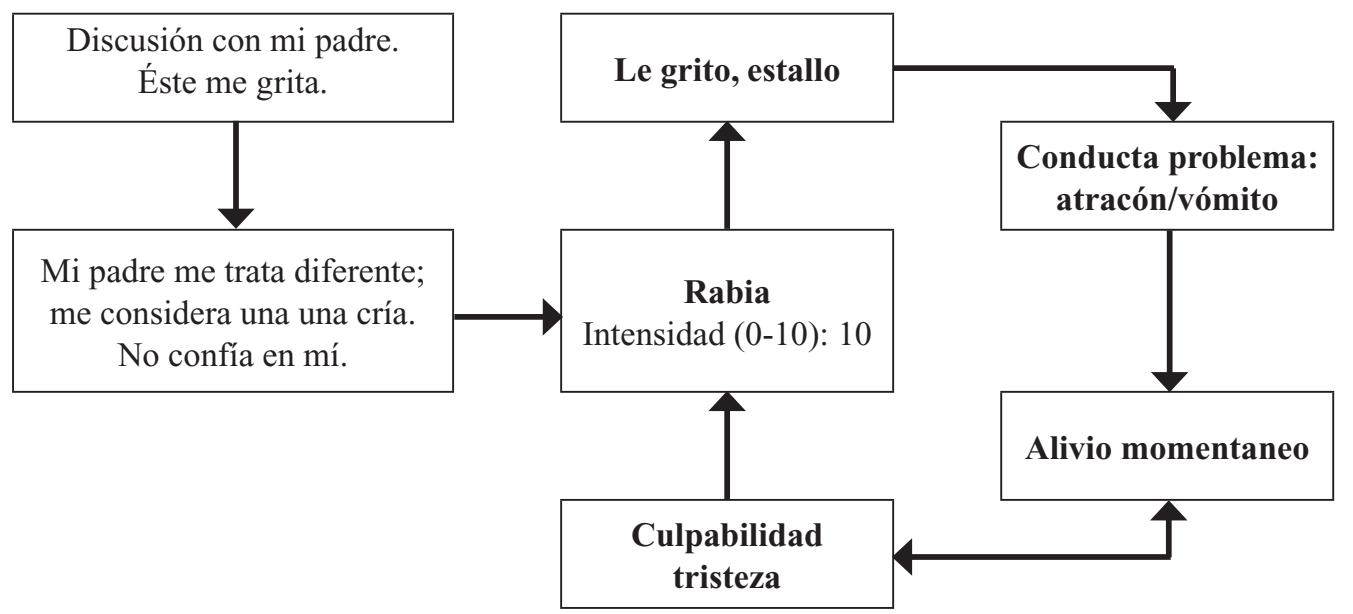

Figura 1. Esquema del análisis conductual del caso

Como podemos observar en este ejemplo, ante una discusión con su padre aparecen pensamientos de que éste no la trata igual que a sus hermanos y de que no confía en ella. Este pensamiento provoca una emoción de rabia que descarga con una conducta agresiva como gritarle. Para evitar ese malestar la paciente se atraca de comida y después vomita. Esta conducta produce un alivio momentáneo a corto plazo, sin embargo, poco después, la paciente se siente culpable y triste por haberlo hecho, lo que incrementa esa sensación de vacío inicial. Este patrón se convierte en un círculo vicioso que se produce cuando ocurre esta situación y se generaliza a situaciones parecidas.

A través de la construcción de análisis funcionales sobre distintas situaciones problemáticas, la paciente fue aprendiendo a identificar los factores de mantenimiento que impedían la resolución del problema. Comenzó a tomar conciencia de la necesidad de aprender a regular las emociones y a sustituir las conductas impulsivas por otras conductas más adaptativas.

Las estrategias utilizadas para tratar las conductas disfuncionales fueron: técnicas de modificación de conducta y validación y potenciación de las estrategias adquiridas por la paciente en terapia grupal.

\section{Terapia grupal}

La terapia grupal consistió en una adaptación del entrenamiento de habilidades en grupo original (Linehan, 1993b). La periodicidad fue de 1 sesión de 2 horas por semana con una pausa de 10 minutos. El grupo constaba de 8 mujeres, 6 de las cuales estaban diagnosticadas de trastorno límite de la personalidad y 2 de rasgos límites. Todas ellas estaban diagnosticadas en el Eje I de un trastorno alimentario. Se decidió incluir a la participante en este grupo puesto que, si bien todavía no cumplía con todos los criterios del trastorno límite, estaba en riesgo de desarrollar el trastorno.

El programa de tratamiento grupal consistió en un entrenamiento en habilidades durante 24 sesiones que incluyeron un módulo inicial de orientación al tratamiento (1 sesión de duración) y cuatro módulos de entrenamiento en habilidades: 1) atención plena (mindfulness) (3 sesiones); 2) regulación emocional (14 sesiones); 3) tolerancia al malestar (2 sesiones); y 4) eficacia interpersonal (4 sesiones). 
Las metas principales del entrenamiento fueron: (1) reducir el desequilibrio interpersonal, emocional, cognitivo, comportamental y las alteraciones de la identidad; (2) aumentar las habilidades de aceptación (habilidades de atención plena y de tolerancia al malestar), y las habilidades de cambio (habilidades de eficacia interpersonal y de regulación emocional).

La paciente recibió 6 meses de terapia grupal. Durante las sesiones se mostró muy motivada y participativa en el grupo. Realizaba con bastante frecuencia las tareas asignadas y compartía con sus compañeras los problemas que había tenido durante la semana.

\section{Procedimiento}

En primer lugar, la terapeuta individual, siguiendo los criterios diagnósticos para los trastornos de personalidad del Eje II del DSM-IV, diagnosticó el TLP mediante la entrevista clínica estructurada SCID-II (First, Gibbon, Spitzer, Williams y Benjamin, 1999). Para delimitar el diagnóstico de trastorno alimentario se utilizó la entrevista clínica de trastornos alimentarios (Perpiñá, Botella y Baños, 2006). Estas entrevistas se realizaron antes de comenzar el tratamiento y fueron administradas en dos sesiones de alrededor de una hora de duración

Antes de comenzar el tratamiento y después del tratamiento, la participante cumplimentó un protocolo de evaluación (evaluación pre-tratamiento) con los instrumentos de autoinforme descritos en el apartado de medidas y los terapeutas rellenaron el inventario de información clínica relevante. Una vez completada la evaluación pre-tratamiento la paciente comenzó el tratamiento (DBT) durante 6 meses (1 sesión semanal de terapia individual de 1 hora, y 1 sesión de terapia grupal semanal de 2 horas). Los resultados de las medidas antes y después del tratamiento fueron comparados. A los seis meses de seguimiento se volvió a administrar el mismo protocolo para observar la evolución de la paciente.

La terapia individual se llevó a cabo por una terapeuta del centro clínico PREVI y la terapia grupal fue dirigida por una terapeuta del Servicio de Asistencia de la Universidad Jaime I. La terapeuta individual adquirió el rol de coterapeuta en la terapia grupal. Ambas terapeutas tienen el grado de doctor, una amplia experiencia en terapia cognitivo-comportamental en diversos trastornos psicológicos y han recibido formación específica en DBT.

\section{RESULTADOS}

Los resultados sobre el estado clínico de la paciente indicaron una mejoría en el funcionamiento global medido por la escala de evaluación de la actividad global (EEAG; DSM-IVTR). Se consiguió mejorar de síntomas graves en el pretratamiento a síntomas moderados y dificultades moderadas en la actividad social, laboral o escolar al final del tratamiento. En el seguimiento el clínico valoró una mejoría adicional: síntomas leves (sólo existe una ligera alteración de la actividad social, laboral o escolar). Por otro lado, se consiguió eliminar el abuso de tóxicos y la frecuencia de vómitos y atracones después del tratamiento. Las conductas sexuales de riesgo también desaparecieron. Estas mejorías se mantuvieron durante el seguimiento.

Tabla 1. Resultados del Inventario Información Clínica Relevante

\begin{tabular}{lccc}
\hline & Pre-tratamiento & Post-tratamiento & Seguimiento \\
Escala de Actividad Global (EjeV) & 50 & 60 & 70 \\
Frecuencia abuso tóxicos/mes & 4 & 0 & 0 \\
Conductas desadaptativas de regulación del afecto: & & 0 & 0 \\
Vómitos/semana & 7 & 0 & 0 \\
Atracones/semana & 7 & 0 & 0 \\
Conductas sexuales de riesgo/semana & 1 & 0 \\
\hline
\end{tabular}


Tabla 2. Puntuaciones en las diferentes variables de autoinforme

\begin{tabular}{lccc}
\hline & Pre-tratamiento & Post-tratamiento & Seguimiento \\
\hline BDI-II & 21 & 6 & 2 \\
STAI-R & 35 & 28 & 10 \\
PANAS-P & 15 & 23 & 33 \\
PANAS-N & 25 & 19 & 12 \\
STAXI-IEI & 47 & 34 & 22 \\
ERQ-R & 16 & 30 & 25 \\
ERQ-S & 18 & 13 & 6 \\
EES & 70 & 50 & 33 \\
RS-15 & 64 & 80 & 91 \\
ICV & 5,1 & 6,9 & 8,7 \\
\hline
\end{tabular}

Nota: BDI-II: Inventario Depresión Beck-Segunda Edición; STAI: Cuestionario Ansiedad Rasgo; PANAS-P: Escala de Afecto Positivo; PANAS-N: Escala de Afecto Negativo; STAXI-IEI: Inventario de Expresión de la ira Estado Rasgo (Indice Expresión y Control de la Ira); ERQ-S: Cuestionario Regulación Emocional (Escala Supresión); ERQ-R: Cuestionario Regulación Emocional (Escala Reevaluación); EES: Escala de Comer Emocional; RS15: Escala de Resilencia; ICV: Índice de Calidad de Vida. Pre-: Evaluación antes del tratamiento. Post-: Evaluación después del tratamiento.

Con respecto a los resultados sobre otros aspectos psicopatológicos después de 6 meses, las puntuaciones indican una disminución de: (1) el BDI-II como medida de depresión (de una puntuación moderada antes del tratamiento, a una puntuación mínima después del tratamiento que se mantiene en el seguimiento); (2) el STAI como una medida de rasgo de ansiedad (antes del tratamiento la puntuación indicaba una elevada ansiedad rasgo y después del tratamiento se encuentra dentro de la normalidad); (3) la subescala de afecto negativo (PANAS-N) (las puntuaciones disminuyen manteniéndose moderadas); (4) el IEI (Índice de Expresión de la Ira) (de una puntuación que indica que el sujeto experimenta intensos sentimientos de ira que pueden ser suprimidos o expresados con conductas agresivas, o producirse ambos hechos, a una puntuación que se interpreta como un comportamiento óptimo después del tratamiento). (4) la subescala de supresión (ERQ-S) del cuestionario de regulación emocional (ambas puntuaciones fueron moderadas en los dos momentos de evaluación); (5) la Escala de Comer Emocional (EES) (se produce una disminución del pretratamiento al postratamiento y en el seguimiento también disminuye).

Asimismo, se produjo un incremento en las puntuaciones de: (1) La subescala de reevalua- ción (ERQ-R) (que se mantiene (aunque atenuada) en el seguimiento). (2) El afecto positivo del PANAS (con puntuaciones moderadas en los dos momentos de evaluación; en el seguimiento se produce una mejoría importante en esta variable). (3) La Escala de Resilencia (RS15) (de una puntuación moderada antes del tratamiento a una puntuación moderadamente alta después del tratamiento y alta en el seguimiento). (4) El Indice de Calidad de Vida (estando las puntuaciones en un rango moderado antes y después del tratamiento, con una mejoría significativa en el seguimiento la paciente puntúa su calidad de vida como alta).

\section{DISCUSIÓN}

Cada vez más los adolescentes constituyen un grupo en el que aparecen rasgos de TLP que puede derivar en un diagnóstico de TLP en la edad adulta (Crawford, et al., 2005). Este hecho hace que la intervención temprana ante la primera presentación de rasgos de TLP sea fundamental.

Podríamos considerar el problema de nuestra paciente como un TLP en desarrollo ya que, si bien no cumple todos los criterios para el diagnóstico de este trastorno (APA, 2000), sí que 
presenta rasgos acusados de TLP. La paciente es una persona adulta, no una adolescente, pero el problema se ha ido complicando progresivamente desde la adolescencia y, en el momento de la evaluación, presenta conductas impulsivas, además de las propias de la bulimia nerviosa, que pueden tener consecuencias muy dañinas como son el abuso de alcohol y las conductas sexuales de riesgo, así como un empeoramiento en áreas de funcionamiento fundamentales como el área interpersonal. Por ello, pensamos que podía ser una buena candidata para explorar de forma preliminar el efecto de la DBT en este perfil de paciente, que todavía no ha desarrollado el cuadro completo de TLP.

Como muestran los resultados, la participante ha respondido extremadamente bien al tratamiento, mejorando en prácticamente todos los aspectos: disminución de las conductas relacionadas con el comportamiento propio del TLP y la patología alimentaria (abuso de sustancias, conductas sexuales de riesgo, comer emocional, vómitos y atracones). Asimismo, encontramos mejorías notables en las medidas relacionadas con la patología de las emociones y regulación del afecto: disminución importante en la sintomatología depresiva y la expresión patológica de la ira y aumento en la utilización de estrategias de regulación emocional a favor de la reevaluación.

Este último dato resulta muy interesante sí tenemos en cuenta que recientes investigaciones han encontrado que la estrategia de reevaluación es una estrategia más adaptativa que la supresión (Gross y John, 2003). Recientemente, se han extendido los trabajos sobre supresión de los pensamientos para examinar la psicopatología específica del TLP. Los hallazgos encontrados sugieren que esta variable puede ser un mediador significativo de intensidad del afecto negativo y reactividad en la sintomatología del TLP (Rosenthal et al., 2008).

Por otro lado, hay que destacar que se consiguió mejorar el nivel de funcionamiento general de la participante, como lo indica el aumento en la escala de evaluación de la actividad global (EEAG) del DSM-IV-TR. Además, encontramos cambios en resiliencia. Aunque hay personas que espontáneamente poseen una mayor capacidad de resiliencia, es un aspecto que se puede desarrollar, y que sirve como factor de pronóstico favorable para superar el trastorno (De Flores Formetí, Lavilla, Molina y López, 2008 , p. 83). Uno de nuestros objetivos es investigar la forma de, no sólo mejorar a nuestros pacientes, sino fortalecerlos, hacerlos más resilientes. Por ello, nos parece un buen resultado que con sólo 6 meses de DBT consigamos un aumento en la resiliencia percibida.

También nos gustaría destacar que estos resultados, nó sólo se mantienen a los seis meses de seguimiento, sino que incluso la paciente mejoró en casi todas las áreas con disminuciones en la sintomatología depresiva, la ansiedad, el afecto negativo, la utilización de las estrategias de supresión para regular el malestar, el comer emocional, y la expresión patológica de la ira; y aumento en autoestima, resiliencia y en el índice de calidad de vida y la escala de actividad global. De hecho, según relata su terapeuta en el informe psicológico del seguimiento: "durante los últimos meses, lleva un funcionamiento muy estructurado con horarios, comidas, horas de sueño, etc. bastante rutinarios que favorecen su estabilidad. Han continuado los contratiempos con su padre pero se maneja de forma adaptativa en estas situaciones. Permanecen rasgos relacionados con relación de dependencia de la pareja, no obstante, el hecho de que vivan en ciudades distintas y lejanas, favorece y contribuye a que la paciente "trabaje" su autonomía y se fortalezcan las relaciones sociales con otras personas".

Los datos nos indican que la DBT ha resultado eficaz globalmente ya que se ha producido una disminución en la sintomatología más grave, es decir, las conductas impulsivas, aspecto clave en el TLP y objetivo fundamental de los creadores de la DBT. Además, hemos encontrado mejorías en otros aspectos relevantes de la patología límite como las emociones y la regulación emocional. Estos resultados son muy alentadores, ya que si se confirma que la DBT es eficaz con pacientes leves, o con pacientes con rasgos acusados de personalidad límite, tendríamos una buena herramienta para prevenir el TLP o para impedir las consecuencias más graves de este trastorno.

Nuestro estudio apoya estos resultados pero constituye una contribución en lo que se refiere 
a una población específica, los pacientes que presentan bulimia nerviosa y rasgos de personalidad límite. En primer lugar, es importante destacar también que, en este caso, la DBT mostró eficacia en aspectos directamente relacionados con la patología de personalidad, pero también en la patología alimentaria, como el comer emocional, las conductas purgativas y los atracones. Nuestros datos van en la línea de los encontrados por otros autores en población anglosajona que han evaluado la eficacia de la DBT para bulimia nerviosa o trastorno por atracón (Safer, Lively, Telch y Agras, 2001; Telch, et al., 2001) y también se asemejan a los datos de un estudio reciente que ha evaluado la eficacia preliminar de la DBT para pacientes con bulimia nerviosa y/o trastorno por atracón y TLP (Chen, Matthews, Allen, Kuo y Linehan, 2008).

Por otro lado, nos gustaría hacer una reflexión sobre la conveniencia de abordar este perfil de pacientes desde una perspectiva más amplia. Pese a que el diagnóstico de eje I (la bulimia nerviosa), está más claro que la patología de personalidad, la formulación del caso de esta paciente nos llevó a determinar que la patología alimentaria estaba muy relacionada con la patología emocional caracterial, por lo que el abordaje se planteó haciendo prioritario el tratamiento de la patología de personalidad y subordinando la patología alimentaria a la patología de personalidad. Los resultados de este estudio de caso, si se replican con otros pacientes, apoyan el abordaje de las recomendaciones de agencias importnantes como el National Institute of Clinical Excellence (NICE, 2004) o la American Psychological Association (APA, 2000).

Con respecto a la intervención temprana, nuestros resultados van en la línea de otros estudios que han adaptado la DBT para pacientes adolescentes (Miller y Rathus 2000) que todavía no habían sido diagnosticados de TLP pero que mostraban algunos síntomas del mismo, con buenos resultados de eficacia (Katz, Gunasekara, y Miller, 2002; Katz et al. 2004; James et al., 2008). Sin embargo, nuestro estudio constituye una contribución en lo que se refiere a una población específica, pacientes que presentan bulimia nerviosa y rasgos de personalidad límite.
Pese a los prometedores resultados de este estudio, debemos ser cautos en la generalización de los mismos y tener presente sus limitaciones. La más importante es que hemos utilizado una metodología de estudio de caso. Pese a las ventajas de esta metodología, las conclusiones que obtenemos son limitadas. Sin embargo, los datos nos animan a seguir trabajando en esta línea ya que la paciente se ha beneficiado de recibir 6 meses de DBT.

\section{BIBLIOGRAFÍA}

American Psychiatic Association (APA) (2000). Diagnostic and statistical manual of mental disorders, revised text (DSM-IV-TR). Whasington, DC: APA.

American Psychiatric Association (2006). Practice guideline for the treatment of patients with eating disorders third edition. Recuperado el 24 de sesptiembre de 2008, desde http://www.psychiatryonline.com/pracGuide/ pracGuideTopic_12.aspx.

Arnow, B., Kenardy, J. y Agras, W.S. (1995). The Emotional Eating Scale: the development of a measure to assess coping with negative affect by eating. The International Journal of Eating Disorder, 18, 79-90.

Beck, A.T., Steer, R.A. y Brown, G.K. (1996). BDI-II. Beck Depression Inventory-Second Edition Manual. San Antonio, TX: The Psychological Corporation.

Bernstein, D.P., Cohen, P., Velez, C.N., et al. (1993). Prevalence and stability of the DSM-III-R personality disorders in a community-based survey of adolescents. American Journal of Psychiatry, 150, 1237-1243.

Bulacio, J.M, Vieyra, M., Daneri, C., Benatuil, D., y Mongiello, E. (2004). Indice de Calidad de Vida: Validación en una muestra argentina. Alcmeon.

Chanen, A.M., Jackson, H. J., McGorry, P.D., Allot, K.A., Clarkson, V. y Yuen, H.P. (2004). Two-year stability of personality disorder in older adolescent outpatients. Journal of Personality Disorders, 18, 526-541.

Chanen, A.M., Jovev, M. y Jackson, H.J. (2007). Adaptive functioning and psychiatric symptoms in adolescents with borderline personality disorder. Journal of Clinical Psychiatry, 68, 297-306.

Chen, E.Y., Matthews, L., Allen, C., Kuo, J. R. y Linehan, M. M. (2008). Dialectical Behaviour Therapy for clients with binge-eating disorder or bulimia nervosa and borderline personality disorder. International Journal of Eating Disorders, 41, 505-512

Conde, V. y Franch, J.I. (1984). Escalas de evaluación comportamental para la cuantificación de la sintoma- 
tología de los trastornos angustiosos y depresivos. Madrid: Upjohn S.A.

Crawford, T.N., Cohen, P., Johnson, J.G., Kasen, S., First, M.B., Gordon, K. y Brook, J.S. (2005). Self-reported personality disorder in the children in the community sample: convergent and prospective validity in late adolescence and adulthood. Journal of Personality Disorders, 19, 30-52.

De Flores Formetí, T., Lavilla, M., Molina, D. y López, B. (2008). Volver a aprender a montar en bicicleta: Trastorno límite de la personalidad. Barcelona: Moral i Torres Editores.

First, M.B., Gibbon, M., Spitzer, R.L., Williams, J.W., y Benjamin, L.S. (1999). Entrevista Clínica Estructurada para los Trastornos de la Personalidad del Eje II del DSM-IV-TR. Barcelona: Masson.

Foa, E.B. y Rothbaum, B.O. (1998). Treating the trauma of rape. New York: Guilford.

García-Palacios, A. (2006). La terapia dialéctico-comportamental: terapia individual. Psicología Conductual, 14, 453-466.

Gross, J.J. y John, O.P. (2003). Individual differences in two emotion regulation processes: Implications for affect, relationships, and well-being. Journal of Personality and Social Psychology, 85, 348-362.

Heilemann, M., Lee, K. y Kury, F.S. (2003). Psychometric properties of the Spanish version of the Resilience Scale. J Nurs Meas, 11, 61-72.

Herzog, D.B., Keller, M.B., Lavori, P. W., Kenny, G.M. y Sacks, N.R. (1992). The prevalence of personality disorders in 210 women with eating disorders. Journal of Clinical Psychiatry, 53, 147-152.

Iglesias, I. y Romero, E. (2009). Estilos parentales percibidos, psicopatología y personalidad en la adolescencia. Revista Española de Psicopatología y Psicología Clínica, 14, 63-77.

Katz, L.Y., Cox, B.J., Gunasekara, S, y Miller, A.L. (2004). Feasibility of dialectical behavior therapy for suicidal adolescent inpatients. Journal of the American Academy of Child and Adolescent Psychiatry, 43, 276-282.

Katz, L.Y., Gunasekara, S., y Miller, A.L. (2002). Dialectical behavior therapy for inpatient and outpatient parasuicidal adolescents. Adolescent Psychiatry, 26, 161-178.

Linehan, M.M. (1993a). Cognitive-Behavioral Treatment of Borderline Personality Disorder. New York: Guilford Press.

Linehan, M.M. (1993b). Skills training manual for treating Borderline Personality Disorder. New York: Guilford Press. Tradución al castellano por Paidós (2003).

Linehan, M., Comtois, K., Murray, A., Brown, M. , Gallop, R., Heard, H., Korslund, K., Tutek, D. Reynolds, S. y Lindenboim, N. (2006). Two-year randomized controlled trial and follow-up of dialectical behavior therapy vs therapy by experts for Suicidal Behaviors and Bor- derline Personality Disorder. Archives of General Psychiatry, 63, 757-766.

Linehan, M.M., Dimeff, L.A., Reynolds, S.K., Comtois, K.A., Welch, S.S., Heagerty, P., y Kivlanhan, D.R. (2002). Dialectical behavioral therapy versus comprehensive validation plus 12-step for the treatment of opioid dependent women meeting criteria for borderline personality disorder. Drug and Alcohol Dependence, 67, 13-26.

Lynch, T.R. (2000). Treatment of elderly depression with personality disorder comorbidity using dialectical behaviour therapy. Cognitive and Behavioural Practice, 7, 468-477.

Lynch, T.R., Chapman, A.L., Rosenthal, M.Z., Kuo, J.R. y Linehan, M.M. (2006). Mechanisms of change in dialectical behavior therapy: theoretical and empirical observations. Journal of Clinical Psychoogy, 62, 459-80.

Matsunaga, H., Kiriike, N., Nagata, T. y Yamagami, S. (1998). Personality disorders in patients with eating disorders in Japan. International Journal of Eating Disorders, 23, 399-408.

Mezzich, J.E., Ruipérez, M.A., Pérez, C., Yoon, G., Liu, J., y Mahmud, S. (2000). The Spanish version of the Quality Of Life Index: presentation and validation. The Journal of Nervous and Mental Disease, 188, 301-305.

Miguel Tobal, J.J., Casado, M.I., Cano Vindel, A., y Spielberger, C.D. (1999). STAXI-2: Inventario de expresión de ira estado-rasgo. Manual $2^{\text {a }}$ Edición Revisada. $P u b$ licaciones de psicología aplicada, $n^{\circ}$. 286. Madrid: TEA Ediciones S.A.

Miller, A.L. y Rathus, J.H. (2000). Dialectical behavior therapy : Adaptations and new applications. Cognitive and Behavioral Practice, 7, 420-425.

National Institute of Clinical Excellence (2004). Eating disorders. Core interventions in the treatment and management of anorexia nervosa, bulimia nervosa, and related eating disorders. Recuperado el 24 de sesptiembre de 2008, desde http://www.nice.org.uk/nicemedia/pdf/CG9FullGuideline.pdf.

Oliva, A., Jiménez, J.M., Parra, A.. y Sánche-Queija (2008). Acontecimientos vitales estresantes, resiliencia y ajuste adolescente. Revista de Psicopatología y Psicología Clínica, 13, 53-62.

Paris, J. (2005). Borderline personality disorder. Canadian Medical Association Journal, 172, 1579-83.

Paris, J. (2009). The treatment of borderline personality disorder: Implications of research on diagnosis, etiology, and outcome. Annual Review of Clinical Psychology, 5, 277-290.

Perpiñá, C., Botella, C. y Baños, R. (2006). Evaluación de los trastornos alimentarios. En V. E. Caballo (Ed.), Manual para la evaluación clínica de los trastornos psicológicos (vol. 2, pp. 211-233). Madrid: Pirámide. 
Rosenthal, M.Z., Gratz, K., Kosson, D. S., Lejuez, C. W., Cheavens, J. S., y Lynch, T.R. (2008). Borderline personality disorder and emotional functioning: A review of the research literature. Clinical Psychology Review, 28, 75-91.

Safer, D.L., Lively, T.J., Telch, C.F., y Agras, W.S. (2001). Dialectical behaviour therapy for bulimia nervosa. American Journal of Psychiatry, 58, 632-634.

Sandín, B., Chorot, P., Lostao, L., Joiner, T. E., Santed, M. A., y Valiente, R.M. (1999). Escalas Panas de afecto positivo y negativo: Validación factorial y convergencia Transcultural. Psicothema, 1, 37-51.

Sanz, J., Navarro, M.E., y Vázquez, C. (2003). Adaptación española del Inventario para la Depresión de Beck-II (BDI-II): 1. Propiedades psicométricas en estudiantes universitarios. Análisis y modificación de conducta, 29 , 239-288.

Spielberger, C.D., Gorsuch, R. L., Lushene, R., Vagg, P.R., y Jacobs, G.A. (1983). Manual for the state-trait anxiety inventory (form y): Self evaluation questionnaire. Palo Alto, CA: Consulting Psychologists Press.

Steiger, H. y Stotland, S. (1996). Prospective study of outcome in bulimics as a function of axis II comorbidity: Long term responses on rating and psychiatric symptoms. International Journal of Eating Disorders, 20, 149-161.

Telch, C.F., Agras, W.S. y Linehan, M.M. (2001). Dialectical behavior therapy for binge eating disorder. Journal of Consulting and Clinical Psychology, 69, 1061-1065.
Van den Bosch, L., Koeter, M., Stijnene, T., Verheul, R. y Van den Brink, W. (2005). Sustained efficacy of dialectical behaviour therapy for borderline personality disorder. Behaviour Research and Therapy, 43, 1231-1241.

Verheul, R., van den Bosch, L., Koeter, M., De Ridder, M.A., Stijnen, T. y van den Brink, W. (2003). Dialectical behavior therapy for women with borderline personality disorder: 12-month, randomised clinical trial in The Netherlands. The British Journal of Psychiatry, 182, 135-140.

Wagnild, G.M. y Young, H.M. (1993). Development and psychometric evaluation of the Resilience Scale. Journal of Nursing Measurement, 1, 165-78.

Watson, D., Clark, L.A. y Tellegen, A. (1988). Development and validation of brief measures of positive and negative affect: the PANAS scales. Journal of Personality and Social Psychology, 54, 1063-70.

Westen, D., Shedler, J., Durrett, C., Glass, S. y Martens, A. (2003). Personality diagnoses in adolescence: DSMIV axis II diagnoses and an empirically derived alternative. Americal Journal of Psychiatry, 160, 952-966.

Wonderlich, S.A. (1995). Personality and eating disorders. En K. D. Brownell y C. G. Fairburn (Eds.), Eating disordes and obesity: A comprehensive handbook. New York: Guilford.

Zanarini, M.C., Frankenburg, F.R., Khera, G.S. y Bleichmar, J. (2001). Treatment histories of borderline inpatients. Comprensive Psychiatry, 42, 144-50. 